\title{
Faktorisierung von Differentialoperatoren.
}

$$
\text { Hermann Dinges }
$$

Wenn eine positive Matrix $P(i, j)$ kleine Elemente hat, im Sinne $\xi P(i, j)<1$ für jedes 1, dann kann I - P faktorisiert werden in eine untere und eine obere Dreiecksmatrix:

$$
I-P=\left(I-P^{-}\right)\left(I-P^{+}\right) \text {. }
$$

Wenn die Indexmenge ein minimales Element hat, kann die Faktorisierung durch iterierte elementare Matrixtransformationen bestimmt werden. Wenn in der Indexmenge eine beliebige Ordnung erklärt ist, wird $\mathrm{P}^{-}$und $\mathrm{P}^{+}$mit Hilfe des durch $\mathrm{P}$ bestimmten Markoffprozesses konstruiert. Die Faktorisierung ist eindeutig bestimmt.

Ist $A$ der infinitesimale Operator einer Diffusion mit genügend regulären Koeffizienten, dann führt eine ähnliche tberlegung auf die Faktorisierung

$$
A=\left(A^{+}+C\right) A^{+}
$$

wo $A^{+}(f) \cdot(x)$ nicht von den Werten $f(y)$ für y mit ergter Koordinate größer als erster Koordinate von $x$ abhängt. $C$ ist ein Differentialoperator erster Ordnung. Die Frage des Bereiches von $A^{+}$ist noch ungeklärt. 